\title{
Approche expérimentale des problèmes vibratoires et acoustiques posés par les pompes rapides. Mise en évidence des phénomènes hydroélastiques
}

\author{
An experimental approach to the vibratory and acoustic problems \\ involved in fast pumps. An examination of the hydro-elastic phenomena
}

\author{
J.C. Guilloud, A. Bonazzi \\ Société Neyrpic, Grenoble
}

B. Garnier

Société Metravib, Lyon
J.C. Bouricot

Etablissements Pompes Guinard

\section{Pourquoi cette étude?}

Dans un très proche avenir, le marché européen des pompes tournant très rapidement va considérablement se développer. Les Etablissements Pompes Guinard envisagent des projets à 12000 et $25000 \mathrm{tr} / \mathrm{mn}$. L'intérêt d'augmenter considérablement la vitesse de rotation tient d'une part au fait que la pompe d'un volume plus réduit coûte moins cher (un impulseur suffit là où il faut aligner une douzaine de roues) et d'autre part, qu'on peut les relier directement à une turbine à gaz, ce quì évite un organe de transmission. Il apparaît évident que le développement de telles pompes nécessite une bonne connaissance des problèmes vibratoires (Metravib a pu constater sur le site de Feyzin les ennuis des pompes américaines Sundyne tournant à $24000 \mathrm{tr} / \mathrm{mn}$ ).

Actuellement, l'utilisateur de pompes tournant avec des vitesses classiques exige que la vitesse nominale soit située en dessous de la première vitesse critique. Cet impératif entraîne le constructeur à concevoir des arbres dont les diamètres sont nettement supérieurs à ceux qui seraient nécessaires pour assurer en toute sécurité le couple d'entrainement. On conçoit que cette approche pose de plus en plus de problèmes avec l'augmentation des vitesses nominales puisque, pour une pression de refoulement imposée, le diamètre des roues centrifuges diminue. Le non-dépassement de la première vitesse critique conduit donc inexorablement, dans le cas des pompes rapides, à un diamètre des roues du même ordre de grandeur que celui de l'arbre qui les porte. Ceci n'est pas réaliste et il devient urgent d'envisager le passage d'une ou de plusieurs vitesses critiques, ce que les constructeurs de très grandes turbines à vapeur -comme par exemple, Alsthom-Atlantique - font couramment. Il est vrai que la prévision a priori de ces vitesses critiques est possible parce que le fluide modifie très peu les caractéristiques vibratoires du rotor.

Pour les pompes très rapides comme pour les "pompes-crayon", on comprend l'intérêt de savoir déterminer les vitesses critiques. Ceci suppose la prise en compte des interactions avec le fluide véhiculé lourd, qui modifient incontestablement les caractéristiques vibratoires et qui sont actuellement très mal connues.

La grande question qui se pose est la suivante : Quelles sont les modifications des caractéristiques vibratoires apportées à un rotor de pompe par le fluide lourd véhiculé?

A plus long terme, se posera le problème d'introduire dans un calcul prévisionnel les termes d'inertie, de raideur et d'amortissement dus à la présence du fluide.

\section{Objectif général de l'étude}

Pour répondre aux questions qui viennent d'être évoquées, Neyrtec Etablissement de Grenoble d'AlsthomAtlantique, l'Etablissement des Pompes Guinard et la Société Metravib ont proposé une approche expérimentale des problèmes hydro-élastiques posés par la détermination des vitesses critiques des machines tournant à très grande vitesse.

Il s'agissait d'une première approche destinée à mettre au point une méthode d'analyse pour déterminer les ordres de grandeur des phénomènes mis en jeu et les classer par ondre d'importance.

Nous n'avions pas promis une interprétation très précise, mais cette phase exploratoire nous a permis de localiser et d'évaluer d'une manière simple certains phénomènes d'inertie ajoutée ainsi que les amortissements dus au fluide véhiculé.

\section{Déroulement de l'étude}

\subsection{Réalisation d'un moyen d'essais}

Ce sont les Etablissements des Pompes Guinard, en accord avec Metravib et Neyrtec, qui ont réalisé une 
pompe à un seul impulseur, ci-après appelée pompemaquette, entrâ̂née par un moteur à fréquence variable permettant de régler et de réguler la vitesse entre 100 et $12000 \mathrm{tr} / \mathrm{mn}$. L'ensemble de ce banc, mis au point chez Guinard, a été installé chez Neyrtec qui fournissait l'infrastructure nécessaire aux essais vibratoires.

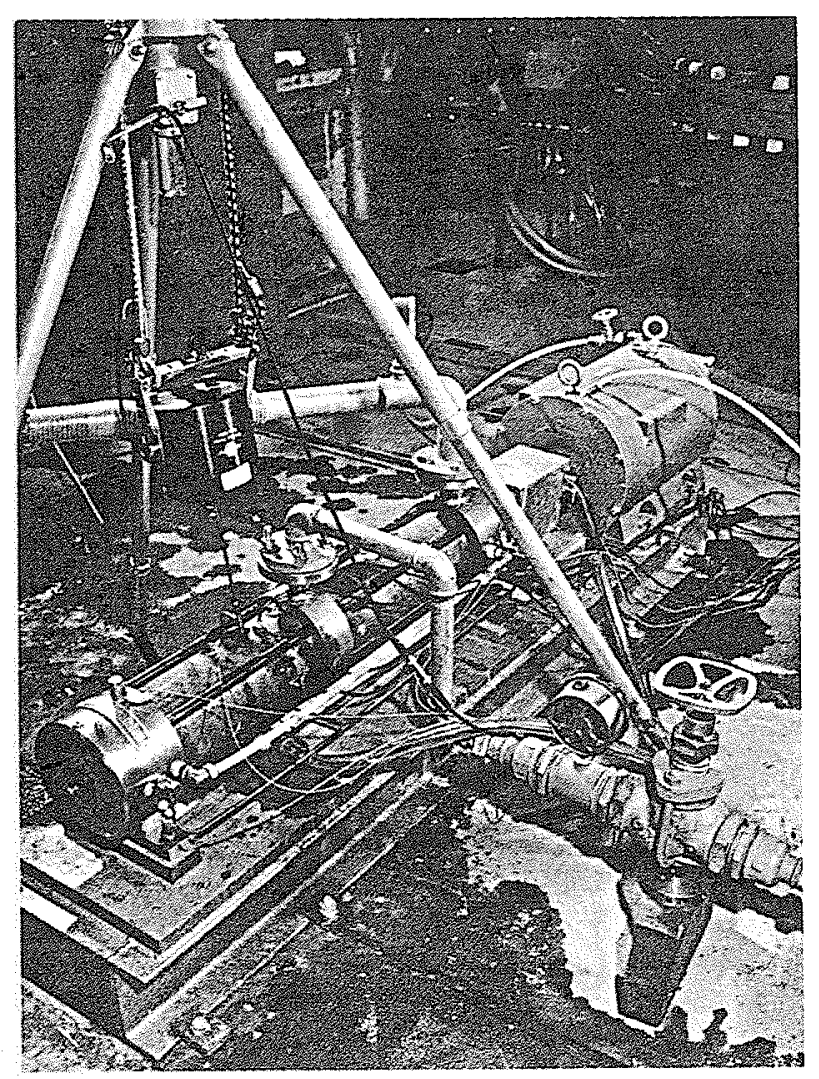

Banc d'essai de pompe rapide.

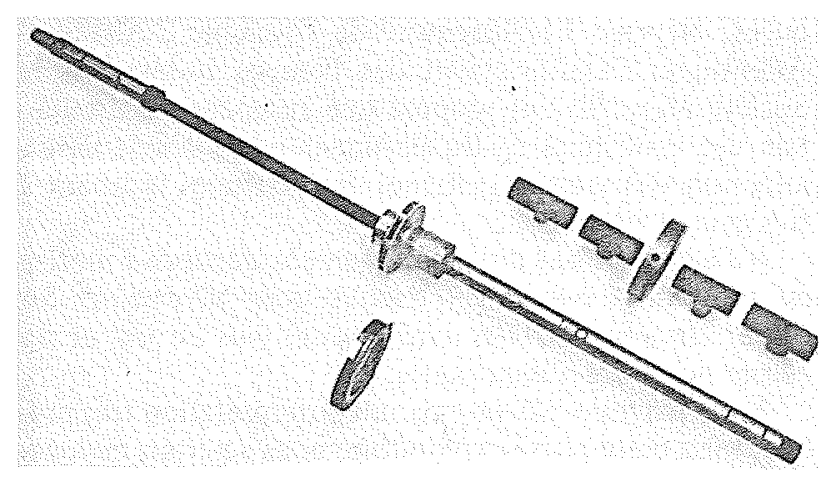

Arbre-pompe, impulseur et masselotte.

Un premier banc d'essai, réalisé avec des paliers hydrostatiques, a dû être abandonné parce qu'il était surdimensionné. La puissance consommée était trop élevée et la seconde vitesse critique était supérieure à $12000 \mathrm{tr} / \mathrm{mn}$. Le moyen décrit ici est donc le second banc construit pour conduire cette étude. Il comprend des paliers à roulements.

\subsection{Définition d'une procédure expérimentale}

Parallèlement à l'étude et à la réalisation du banc d'essais, Neyrtec et Metravib ont défini une procédure expérimentale de mise en évidence des effets hydroélastiques propres à modifier les comportements vibratoires déjà connus d'un rotor évoluant dans le vide ou dans un fluide léger n'apportant que peu d'interactions.

Cette analyse expérimentale est basée sur le principe suivant :

Caractériser les effets hydro-élastiques en comparant les résultats vibratoires de la pompe en rotation dans l'eau autour des deux premières vitesses critiques à ceux obtenus en flexion pure, le rotor étant alors arrêté et noyé ou non dans l'eau "immobile" de la pompe.

Il s'agit donc bien d'une approche globale de l'objectif général annoncé, seule solution qui convenait pour cette phase initiale destinée à appréhender les principaux phénomènes et ordres de grandeur.

\subsection{Essais de vibration}

Les expériences vibratoires ont été essentiellement conduites par Metravib à l'aide de son laboratoire mobile d'analyse des vibrations selon la procédure suivante :

a) Analyse modale du rotor libre-libre dans l'air puis dans l'eau d'une grande cuve (milieu liquide non confiné).

b) Analyse modale des vibrations du banc d'essai rotor enlevé (enveloppe externe de la pompe et moteur fixés à un bâti posé sur un bloc de béton)

c) Etude des premiers modes de flexion du rotor monté dans la pompe en l'absence d'eau (essais qualifiés dans la suite de rotor monté en air)

d) Analyse modale de la flexion du rotor monté dans la pompe avec de l'eau. L'absence de rotation nous a permis de qualifier ces essais de rotor monté en eau.

e) Etude des deux premières vitesses critiques, la pompe étant maintenue en rotation critique pendant le temps nécessaire pour faire l'acquisition des données.
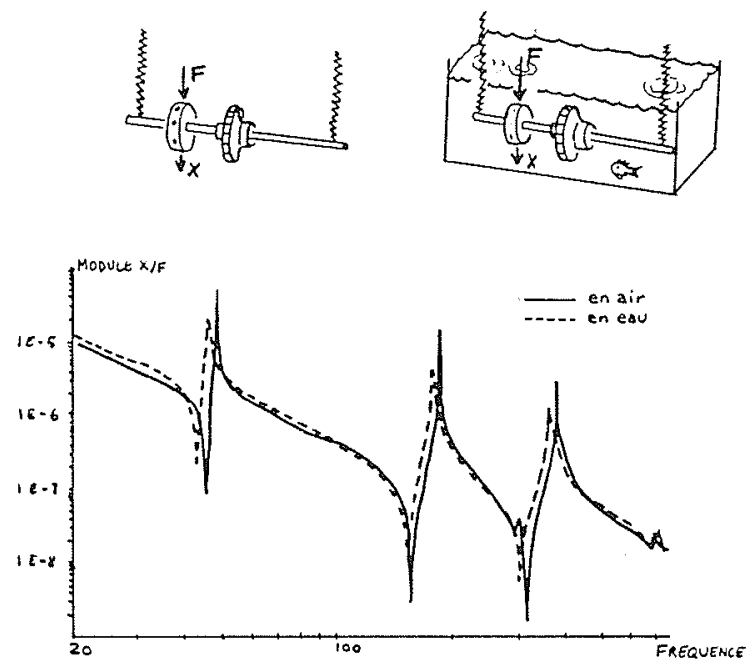

Figure 1. - Effets de l'eau en milieu non confiné sur le rotor libre-libre. 


\begin{tabular}{|c|c|c|c|c|c|}
\hline \multirow{3}{*}{ Mode } & \multirow{3}{*}{ Conditions } & \multicolumn{2}{|c|}{ Première expérience } & \multicolumn{2}{|c|}{ Deuxième expérience } \\
\hline & & \multicolumn{2}{|c|}{ rotor monté } & \multicolumn{2}{|c|}{ rotor affaibli } \\
\hline & & en air & en eau & en air & en eau \\
\hline 1 & $\begin{array}{c}\text { fréquence } \\
\text { amortissement } \\
\text { souplesse }\end{array}$ & $\begin{array}{c}56,52 \\
0,11 \\
1,92.10^{-6}\end{array}$ & $\begin{array}{cc}38,88 & (-31 \%) \\
0,40 & (+0,29) \\
2.10 \cdot 10^{-6}\end{array}$ & $\begin{array}{c}37,17 \\
0,154 \\
3,79.10^{-6}\end{array}$ & $\begin{array}{lr}27,8 & (-33 \% \\
0,476 & (+0,32) \\
3,68 \cdot 10^{-6}\end{array}$ \\
\hline 2 & $\begin{array}{c}\text { fréquence } \\
\text { amortissement } \\
\text { souplesse }\end{array}$ & $\begin{array}{c}219,4 \\
0,043 \\
4,22 \cdot 10^{-8}\end{array}$ & $\begin{array}{cc}207,85 & (-5,3 \%) \\
0,045 & (+0,002) \\
5,39 \cdot 10^{-8}\end{array}$ & $\begin{array}{c}132-151 \\
\text { moda } 2 \text { dédoublé }\end{array}$ & $\begin{array}{c}129-146 \\
(-2,3 \%) \quad(-3,4 \%)\end{array}$ \\
\hline 3 & $\begin{array}{c}\text { fréquence } \\
\text { amortissement } \\
\text { souplesse }\end{array}$ & $\begin{array}{c}308,7 \\
0,051 \\
2,27.10^{-8}\end{array}$ & $\begin{array}{cr}284,50 & (-7,8 \%) \\
0,069 & (+0,018) \\
2,75 \cdot 10^{-8} & \\
\end{array}$ & $\begin{array}{c}221-225 \\
\text { mode } 3 \text { dédoublé }\end{array}$ & $\begin{array}{c}195-225 \\
(-13 \%) \quad(-13 \%)\end{array}$ \\
\hline
\end{tabular}

\section{Principaux résultats}

\subsection{Effet de l'eau dans le corps de pompe}

Toutes choses égales par ailleurs, on compare les fréquences propres de l'arbre monté dans la pompe selon que cette dernière est vide ou pleine d'eau, le rotor ne tournant pas.

On dispose là de deux séries parallèles d'expériences (rotor initial et rotor affaibli). L'effet de l'eau, s'il diffère beaucoup du cas où le rotor est placé en milieu
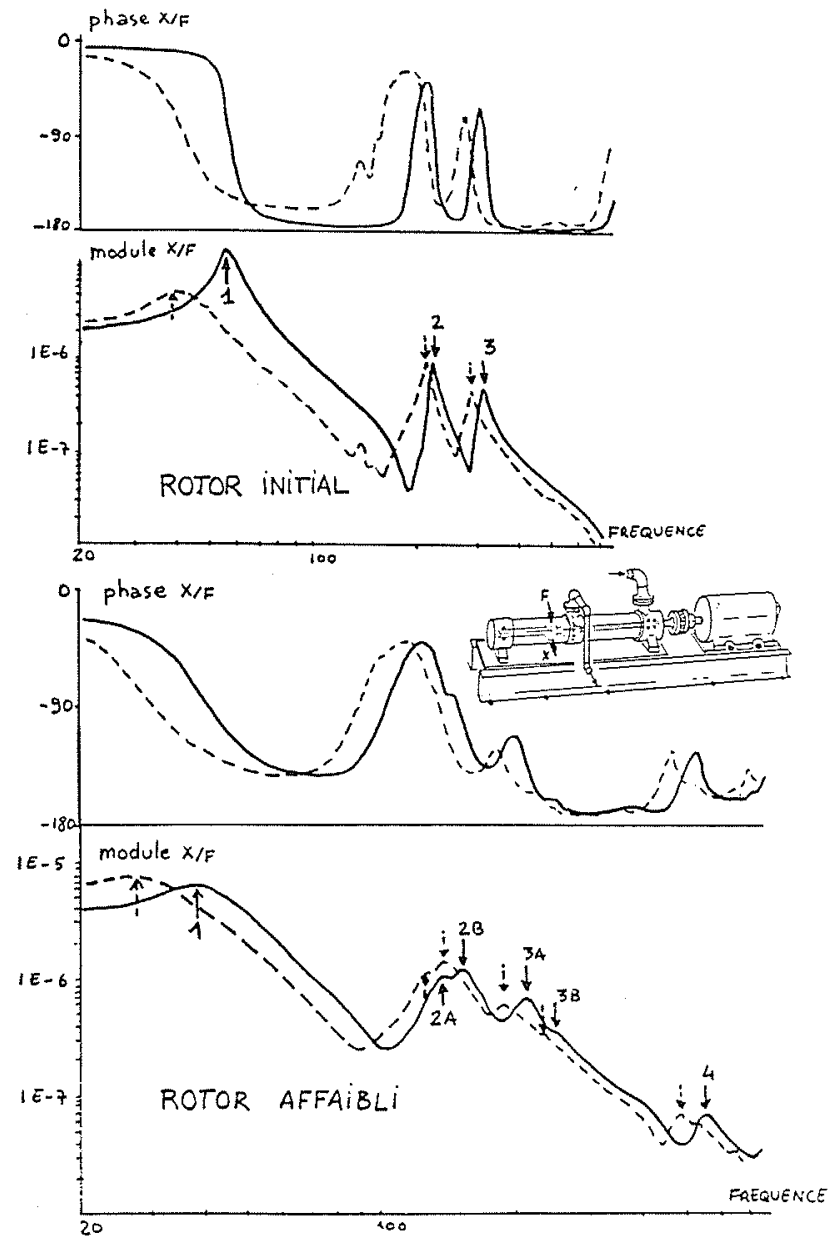

Figure 2. - Effets de l'eau sur les modes de l'arbre à l'arrêt dans le corps de pompe. infini (voir figure 1), est tout à fait semblable dans les deux séries, et surtout très variable d'un mode à l'autre (Fig. 2). L'effet prépondérant est donc lié à la forme propre de l'arbre au niveau des zones les plus confinées entre rotor et bâti. Les effets les plus marquants apparaissent sur le premier mode :

- fréquence propre très abaissée (plus de $30 \%$ de glissement);

- amortissement considérable ( $\operatorname{tg} \delta=2 \xi$ accru de $30 \%$ environ).
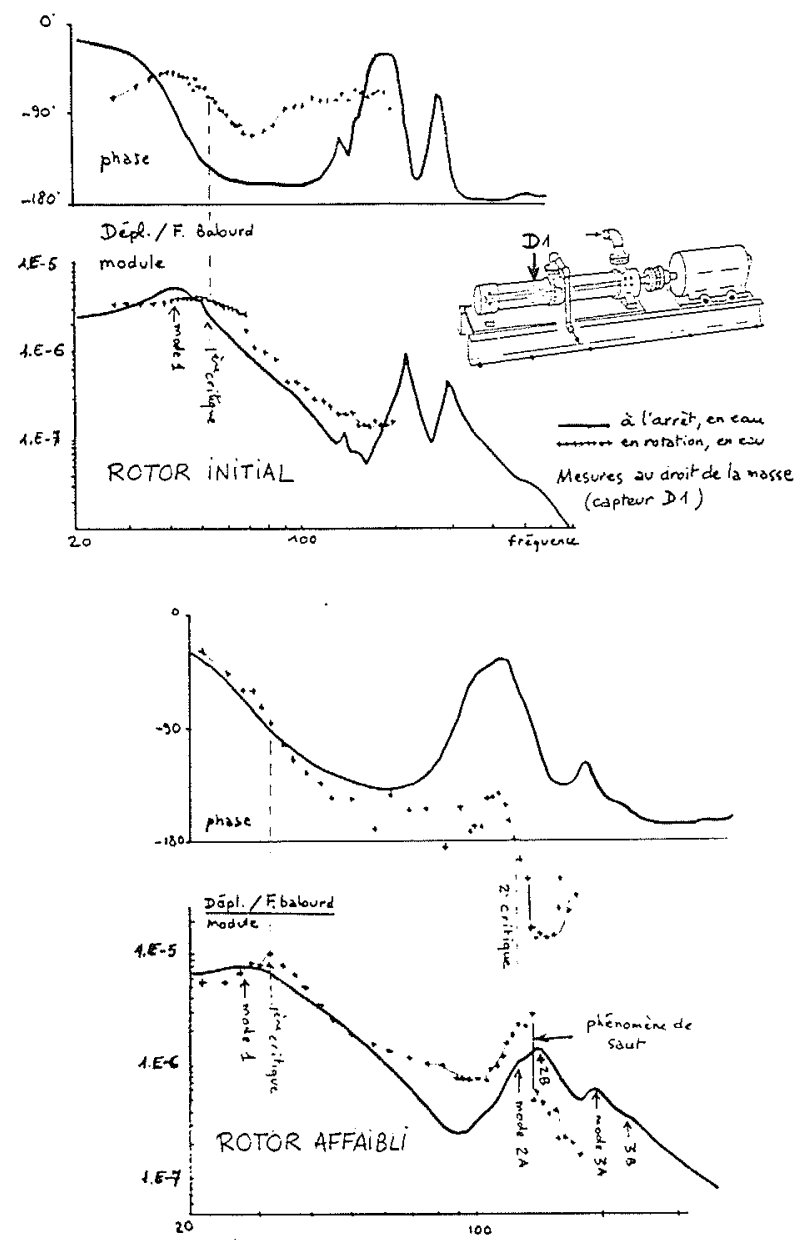

Figure 3. - Essais en rotation en eau. Comparaison avec impédances à l'arrêt en eau. 
Ils sont par contre minimes sur le(s) deuxième(s) mode $(s)$, un peu plus sensibles sur le $(s)$ troisième $(s)$ $\operatorname{mode}(s)$.

\subsection{Essais en rotation}

Le second résultat important est que, mis à part les effets gyroscopiques, les vitesses critiques ne coincident pas avec les fréquences propres de flexion du rotor dans l'eau (Fig. 3). Si la diminution des fréquences propres de flexion du rotor immergé dans la pompe s'explique très bien par les effets d'inertie ajoutée par le fluide dans certaines zones de faibles jeux entre le rotor et le corps de la pompe, nous n'avons pas pu réussir dans cette phase exploratoire à expliquer le fait que les vitesses critiques sont supérieures aux fréquences propres de flexion du rotor dans l'eau. On notera le fait important qu'une pompe peut très bien tourner sur certaines vitesses critiques sans que l'on puisse s'en apercevoir. Cette circonstance tient à l'existence d'amortissements élevés dus au fluide véhiculé dans les mêmes zones de faibles jeu où se manifestent les effets d'inertie ajoutée.

\section{Conclusions}

Bien que cette étude ait eu pour objectif l'amélioration des connaissances de certains effets hydro-élastiques, on conçoit son intérêt pour réduire le bruit rayonné par un circuit muni d'une pompe rapide en examinant la figure 4 . Bien qu'il existe des amortissements importants dans une pompe, il ne faut, en aucune façon, que sa vitesse de rotation soit voisine des fréquences critiques du rotor et du bâti. Pour prédire dès le stade de la conception et éviter cette confusion des fréquences où

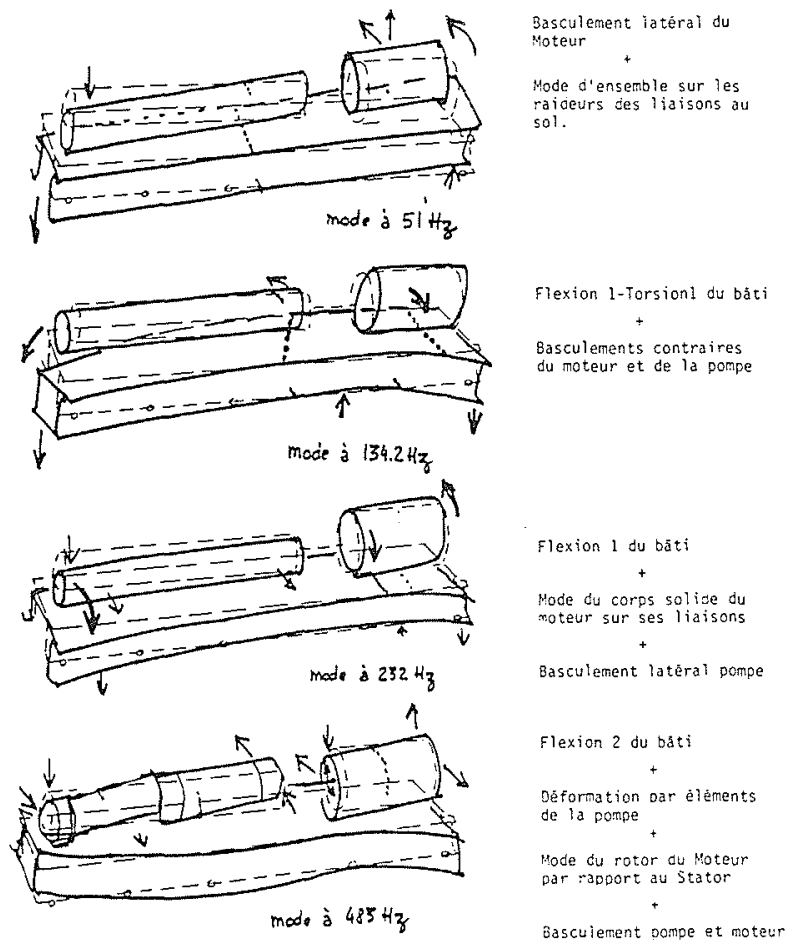

Figure 4. - Principales déformées du bati compte tenu du circuit d'alimentation de la pompe.

l'émission sonore est renforcée, il faut être mâitre des interactions hydro-élastiques entre le rotor et le reste de toute la structure.

On remarque que l'effet de l'eau est assez homogène sur les divers modes (chute de la fréquence de l'ordre de $5 \%$, l'amortissement supplémentaire décroissant régulièrement avec l'ordre du mode de $7,5 \%$ à $1,7 \%$ ). 